\title{
The burden of celiac disease in Canada: More work needed to lighten the load
}

\author{
John K Marshall MD MSc FRCPC AGAF
}

$\mathrm{C}$ eliac disease was first recognized as a distinct clinical entity more than 60 years ago. However, its full spectrum and impact have only been appreciated over the past decade. In part, this reflects the increasing availability of serological testing that has enabled earlier diagnosis, and a greater appreciation of the protean clinical manifestations and high prevalence of gluten sensitivity.

In the current issue of the Canadian Journal of Gastroenterology, Pulido et al (1) (pages 449-453) surveyed the memberships of the Canadian Celiac Association and the Fondation Quebecoise de la Maladie Coeliaque to characterize a Canadian adult population with celiac disease. Remarkably, 5912 of 10,693 invitees responded. Key findings included an average diagnostic delay of 12.0 years and a high prevalence of persistent symptoms despite the avoidance of gluten. Methodological shortfalls, however, must be acknowledged. Most notably, the retrospective survey design is prone to both response bias and recall bias, and members of national celiac organizations represent a highly selected subset of the overall population with celiac disease. Nonetheless, these data provide useful insight into the symptom burden and clinical challenges faced by patients with celiac disease.

To some extent, delayed diagnosis of celiac is understandable, given that 'classic' presentations are now rare and many patients initially present with extraintestinal signs such as anemia. However, diagnostic delay can have important health consequences that range from persistent symptoms to micronutrient deficiencies and even malignancies. There is ample evidence that celiac disease remains underdiagnosed in Western populations and that its incidence is increasing (2). What is the solution? Population-based screening remains controversial; however, better education of health care providers and proactive screening of those at increased risk could help. In Canada, more consistent access to serological screening assays is needed because not all provincial health ministries reimburse the test.

The primary treatment for celiac disease is elimination of gluten from the diet on diagnosis (3). For most patients, this is highly effective, but persistent symptoms are common, as demonstrated by Pulido et al (1). There are three potential explanations for such therapeutic failure. Some patients have symptoms unrelated to gluten sensitivity, and other competing etiologies must be investigated. Other patients may either surreptitiously or unintentionally continue to ingest gluten, which mandates a careful review of diet and other environmental exposures. However, the most challenging scenario is that of refractory celiac disease, for which novel approaches are needed.

Nonresponsive celiac disease is classified as either type I, in which duodenal lymphocytic infiltration resembles untreated disease, or type II, in which lymphocytes carry an abnormal immunophenotype with oligoclonal expansion. The prognosis of the latter is less favourable. The traditional medical approach to such patients has included corticosteroids and immunosuppression. However, a number of novel and targeted approaches to nonresponsive disease are on the horizon. For example, larazotide acetate is an oral peptide that affects tight junction function that has shown promise in phase II placebo-controlled trials (4). Oral endopeptidases that convert residual dietary gluten to nontoxic peptides are in clinical trials (5) and preventive vaccines have been proposed. In animal models, a copolymer of hydroxyethyl methacrylate and styrene sulfonate appears to prevent digestion of dietary gluten to toxic peptides (6). Of course, probiotics are also being explored to modulate intestinal immune responses (7).

Despite promise for the future, celiac disease remains a lifelong diagnosis that requires ongoing multidisciplinary care and careful longitudinal review. Both gastroenterologists and primary care practitioners play important roles in monitoring adherence and detecting the metabolic consequences of micronutrient deficiency. However, involvement of a skilled dietitian, both for initial instruction and for ongoing review of dietary adherence, is critical to treatment success. Support groups such as the Canadian Celiac Association (www. celiac.ca) provide valuable resources for patients, families and caregivers. Effective advocacy from such organizations has contributed to the enhanced availability of reliably gluten-free alternatives throughout the hospitality, grocery and transportation industries in Canada.

Pulido et al (1) have helped to shed light on the burden of illness of celiac disease in Canada. While the past decade has witnessed great progress in the detection, support and management of people living with celiac disease, much work remains. These and similar observations from other jurisdictions call for the investment of the funds, effort and infrastructure required to eliminate the burden of celiac disease altogether.

\section{REFERENCES}

1. Pulido O, Zarkadas M, Dubois S, et al. Clinical features and symptom recovery on gluten-free diet in Canadian adults with celiac disease. Can J Gastroenterol 2013:27:449-53.

2. Ludvigsson JF, Rubio-Tapia A, Van Dyke CT, et al. Increasing incidence of celiac disease in a North American population. Am J Gastroenterol 2013;108:818-24.

3. Rubio-Tapia A, Hill ID, Kelly CP, Calderwood AH, Murray JA. ACG clinical guidelines: Diagnosis and management of celiac disease. Am J Gastroenterol 2013;108:656-76.

4. Kelly CP Green PH, Murray JA, et al. Larazotide acetate in patients with celiac disease undergoing a gluten challenge: A randomized placebo-controlled study. Aliment Pharmacol Ther 2013;37:252-62.

5. Siegel M, Garber ME, Spencer AG, et al. Safety, tolerability and activity of ALV003: Results from two phase I single, escalating dose trials. Dig Dis Sci 2012;57:440-50.

6. Pinier M, Fuhrmann G, Galipeau HG, et al. The copolymer $\mathrm{P}(\mathrm{HEMA}-\mathrm{co}-\mathrm{SS})$ binds gluten and reduces immune response in gluten-sensitized mice and human tissues. Gastroenterology 2012;142:316-25.

7. Smecuol E, Hwant HJ, Sugai E, et al. Exploratory randomized double-blind placebo-controlled study on the effects of Bifidobacterium infantis natren life start strain super strain in celiac disease. J Clin Gastroenterol 2013;47:139-47.

McMaster University, Hamilton, Ontario

Correspondence: Dr John K Marshall, Division of Gastroenterology (2F59), McMaster University, 1280 Main Street West, Hamilton,

Ontario L8S 4K1. Telephone 905-521-2100 ext 76782, fax 905-523-6048, e-mail marshllj@mcmaster.ca

Received and accepted for publication July 8, 2013 


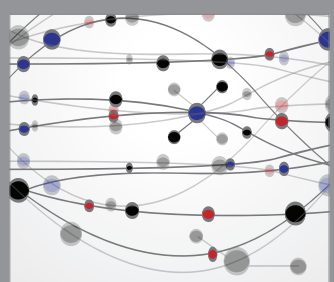

The Scientific World Journal
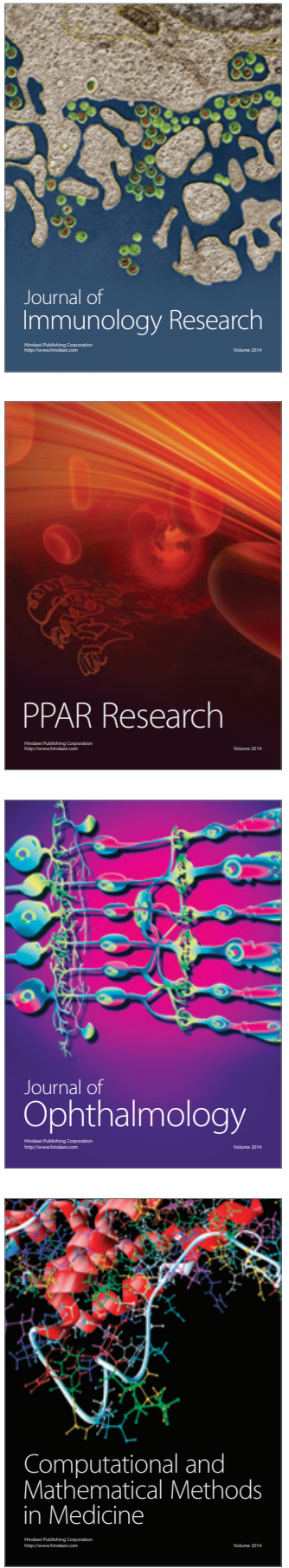

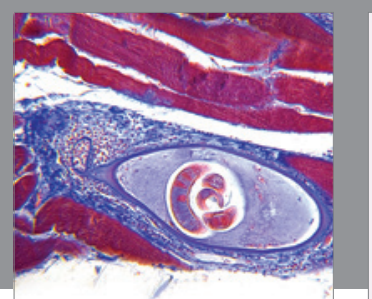

Gastroenterology Research and Practice

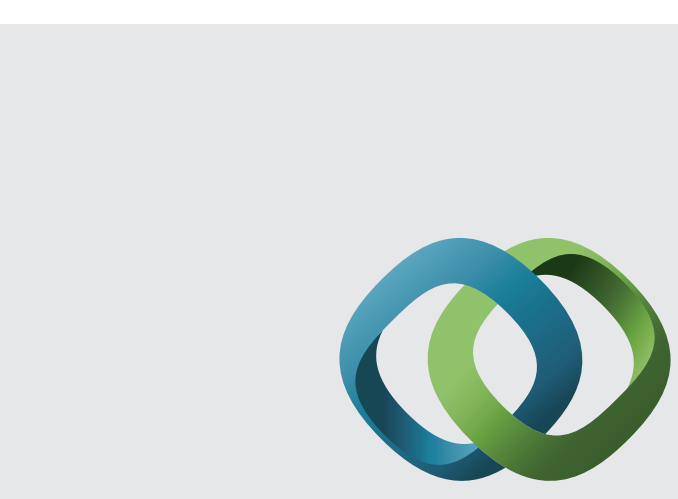

\section{Hindawi}

Submit your manuscripts at

http://www.hindawi.com
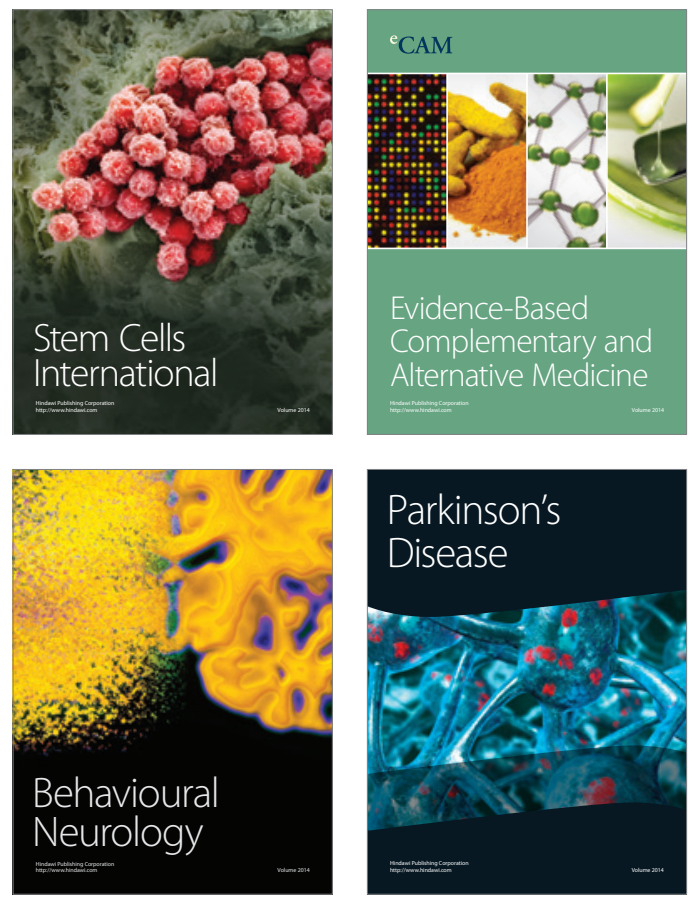
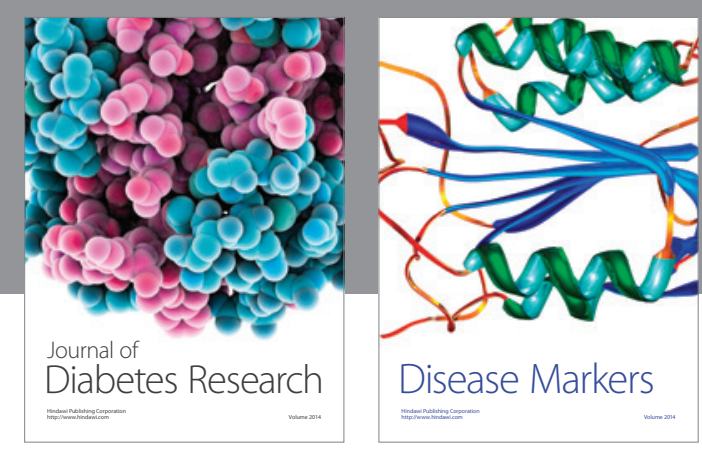

Disease Markers
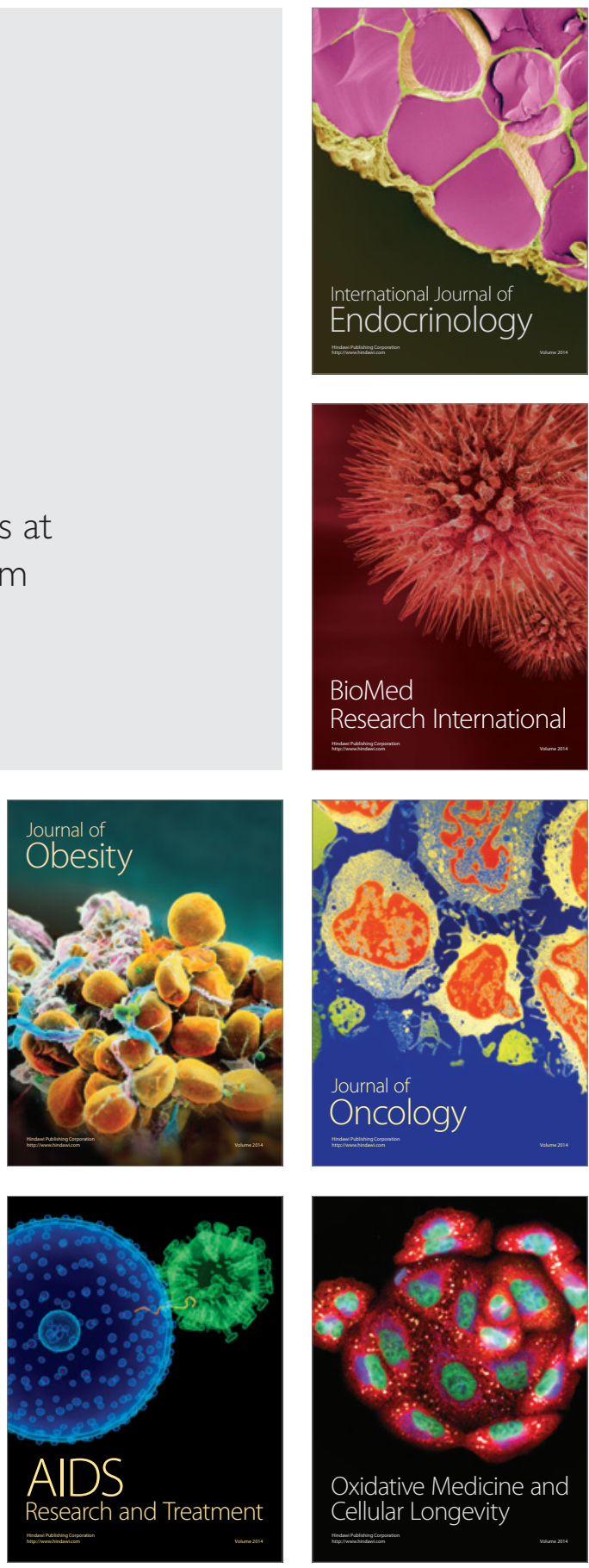